\title{
Ion acceleration from microstructured targets irradiated by high-intensity picosecond laser pulses
}

\author{
M. Bailly-Grandvaux $\odot,{ }^{1,}{ }^{*}$ D. Kawahito $\odot,{ }^{1}$ C. McGuffey $\odot,{ }^{1}$ J. Strehlow,${ }^{1,2}$ B. Edghill,,${ }^{1,2}$ M. S. Wei, ${ }^{3}$ N. Alexander, ${ }^{4}$ \\ A. Haid, ${ }^{4}$ C. Brabetz ${ }^{\circ},{ }^{5}$ V. Bagnoud, ${ }^{5}$ R. Hollinger $\odot,{ }^{6}$ M. G. Capeluto $\odot,{ }^{6,7}$ J. J. Rocca, ${ }^{6}$ and F. N. Beg $\oplus^{1,2}$ \\ ${ }^{1}$ Center for Energy Research, University of California San Diego, La Jolla, California 92093, USA \\ ${ }^{2}$ Department of Mechanical and Aerospace Engineering, University of California San Diego, La Jolla, California 92093, USA \\ ${ }^{3}$ Laboratory for Laser Energetics, Rochester, New York 14623, USA \\ ${ }^{4}$ General Atomics, San Diego, California 92121, USA \\ ${ }^{5}$ GSI Helmholtzzentrum für Schwerionenforschung, Darmstadt 64291, Germany \\ ${ }^{6}$ Physics Department, Colorado State University, Fort Collins, Colorado 80523, USA \\ ${ }^{7}$ Departamento de Física, FCEyN, UBA and IFIBA, CONICET, 1428 Buenos Aires, Argentina
}

(Received 13 April 2020; accepted 1 July 2020; published 3 August 2020)

\begin{abstract}
Structures on the front surface of thin foil targets for laser-driven ion acceleration have been proposed to increase the ion source maximum energy and conversion efficiency. While structures have been shown to significantly boost the proton acceleration from pulses of moderate-energy fluence, their performance on tightly focused and high-energy lasers remains unclear. Here, we report the results of laser-driven three-dimensional (3D)-printed microtube targets, focusing on their efficacy for ion acceleration. Using the high-contrast $\left(\sim 10^{12}\right)$ PHELIX laser $\left(150 \mathrm{~J}, 10^{21} \mathrm{~W} / \mathrm{cm}^{2}\right)$, we studied the acceleration of ions from $1-\mu \mathrm{m}$-thick foils covered with micropillars or microtubes, which we compared with flat foils. The front-surface structures significantly increased the conversion efficiency from laser to light ions, with up to a factor of 5 higher proton number with respect to a flat target, albeit without an increase of the cutoff energy. An optimum diameter was found for the microtube targets. Our findings are supported by a systematic particle-in-cell modeling investigation of ion acceleration using 2D simulations with various structure dimensions. Simulations reproduce the experimental data with good agreement, including the observation of the optimum tube diameter, and reveal that the laser is shuttered by the plasma filling the tubes, explaining why the ion cutoff energy was not increased in this regime.
\end{abstract}

DOI: 10.1103/PhysRevE.102.021201

\section{INTRODUCTION}

Ion sources based on high-intensity short pulse lasers stand out from conventional sources because they have unique benefits, including high current and brightness, potentially tunable ion energy spectral shape and energies, and low emittance from a compact and relatively inexpensive platform. It is worth mentioning though that the size and cost of a laser system strongly increases with the amount of laser energy it can deliver in one shot. When directed into a target, the laser-driven ion beam can create conditions as extreme as the interior of planets and stars (pressures, temperatures, densities, etc.) through intense, rapid energy deposition. This aspect is crucial to many high-impact applications [1-3], such as laboratory astrophysics [4], geophysics [5], ion fast ignition for fusion [6], ion oncology [7-9], and isotope production and neutron generation [10]. The short bunch duration proton beam can also be used to probe various ultrafast phenomena in high-energy-density laboratory plasmas [11-16].

Significant development of laser ion sources has been conducted with chirped-pulse amplified laser systems delivering short (30-700 fs) laser pulses with peak intensities in the range of $10^{20}-10^{22} \mathrm{~W} / \mathrm{cm}^{2}$. Multiple performance metrics for the aforementioned applications improve as target thickness

\footnotetext{
*mbaillygrandvaux@ucsd.edu
}

is reduced and intensity is increased, leading to a higher density and temperature of electrons at the rear side of the target. The electron density, for example, strongly scales with the laser intensity and is inversely proportional to the square of the target thickness $[17,18]$. In the target normal sheath acceleration mechanism [19], the total electron energy density at the rear of the target is the fundamental quantity that determines the ion cutoff energy.

Thin targets with engineered surface structures have garnered increasing attention as a possible means to increase laser-to-ion conversion efficiency. The gains expected by using thin (micron-thick) target foils and front-surface structures on the scale of the laser wavelength require ultrahigh contrast $\left(>10^{11}\right)$ for the laser intensities typically applied $\left(10^{20}-10^{22} \mathrm{~W} / \mathrm{cm}^{2}\right)$ to avoid damage of such surface structures prior to the main pulse interaction [20,21]. When a laser pulse irradiates a microstructure, electrons from the structure are pulled out into the laser field and accelerated [22]. Generally speaking, electrons are an intermediary in ion acceleration mechanisms, so that gains in the conversion efficiency to electrons can be expected to carry over to ions. For example, the electron bunches can become separated periodically by one laser wavelength, and electrons in appropriate phases copropagate with the driving laser and reinforce the subsequent conversion efficiency to ions [23-25]. A second effect, especially with waveguiding structures such as microtubes, is light intensification. With wavelength-scale structures, the 
laser field is redistributed about the target surfaces due to diffraction, leading to light intensification in the near-field area and thus triggering higher on-target intensities compared to flat targets $[26,27]$.

There have been several demonstrations of enhanced electron and ion energy from microstructured targets, both experimentally [22,24,28-33] and numerically [25-27,3437]. In addition, structured targets composed of nanopillars or nanolayers have been shown to yield a higher conversion of the laser energy to protons, neutrons, and electrons $[22,23,38]$, and high- $Z$ wires have led to improvements for X-ray [21,23,39-43] and terahertz sources [44]. However, previous ion acceleration experiments with microstructured targets were made at moderate laser fluence, using either ultrashort pulses $(<100 \mathrm{fs})$ with moderate laser energy $(\leqslant 10 \mathrm{~J})$ $[22,24,28,29,31]$ or defocused beams [30]. The latter experiment was conducted on the same laser as presented here but defocused to a lower intensity $\left(I=10^{17-18} \mathrm{~W} / \mathrm{cm}^{2}\right)$. In the aforementioned studies, the laser energy fluence on the target was $\ll 1 J / \mu \mathrm{m}^{2}$, which limits the expansion of the structures during the main pulse. At higher fluence there are important implications of expansion on coupling and ion acceleration which we will discuss in this Rapid Communication.

Recently, Sedov et al. [32] reported the features of electron and ion acceleration from nanowire targets irradiated by a high-intensity picosecond laser pulse, in a regime similar to our experiment. The authors witnessed an enhancement in laser absorption via x-ray measurement, although neither an increase of the accelerated ion number nor cutoff energies were observed. The authors attribute the lower acceleration field to the larger volume of the relief target, leading to a lower hot-electron density. We believe that this effect is actually a direct consequence of the structure's expansion during the main pulse. The authors expressed interest in a future investigation of the trends with structure dimensions. In the last two works mentioned, the critical density contour is key, where $n_{c} \approx 10^{21} \lambda_{\mu \mathrm{m}}^{-2} \mathrm{~cm}^{-3}$ with $\lambda_{\mu \mathrm{m}}$ being the laser wavelength in units of $\mu \mathrm{m}$.

This Rapid Communication presents a study on the effect of microstructures added to otherwise standard targets upon ion acceleration for a high-energy laser with short focusing, in a regime typical of today's frontier ion acceleration experiments. We report proton and carbon beam spectra from the microstructured targets of different geometries and dimensions including microtube-type targets. Although microtube targets have been studied previously using PIC codes [26,27], this work presents both experimental and simulation results of ion acceleration with microtube targets. An optimal structure dimension was identified that resulted in a strong enhancement of proton and carbon acceleration.

Here, we show that the total numbers of protons and carbons were increased relative to a flat reference by a factor up to $\approx 5 \times$ for protons and $\approx 2 \times$ for carbons. Correspondingly, the energy conversion efficiency from the laser to accelerated protons increased by a factor of nearly $3 \times$, from $\approx 3 \%$ (flat foil) to $\approx 7.5 \%$. Yet, no clear enhancements of the ion cutoff energies were observed. We show further that the results, including the optimal dimension, are explained by two-dimensional (2D) particle-in-cell (PIC) simulations. Moreover, the simulations showed that the engineered struc-

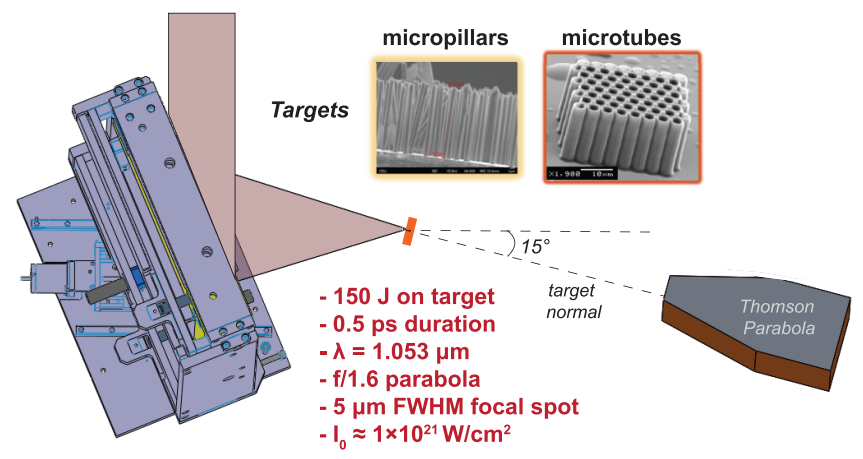

FIG. 1. Sketch of the experimental setup: The PHELIX laser was focused with $15^{\circ}$ incidence onto flat and microstructured $1-\mu \mathrm{m}$ foils. Accelerated ions were detected along the target normal with a Thomson parabola spectrometer. The inset images show microscope images of two structure types.

tures expanded rapidly and shuttered the laser around the time of its peak magnitude, which explains why the ion cutoff energy was not increased in this regime.

\section{EXPERIMENTAL SETUP}

The experiment was conducted at the high-contrast $\left(\sim 10^{12}\right)$ PHELIX laser system, delivering $150 \mathrm{~J}$ on target in $500 \mathrm{fs}$, focused with an $f / 1.6$ parabola down to a $5-\mu \mathrm{m}$ full width at half maximum (FWHM) focal spot, reaching a peak intensity of $\sim 1 \times 10^{21} \mathrm{~W} / \mathrm{cm}^{2}$ with vacuum focus. The laser incidence angle was $15^{\circ}$ as required by the facility at this level of laser energy to avoid unwanted light backscattering to the laser chain. The setup is sketched in Fig. 1. The laser was focused onto $\mathrm{CH}$ microtube arrays or Co micropillars extending off one side of a 1- $\mu$ m-thick Co substrate. Flat reference targets were also studied for comparison and consisted of only the substrate layer with the same material and thickness. The accelerated ion energy distribution was measured along the target normal using a Thomson parabola (TP) spectrometer [45], operating at a voltage of $8 \mathrm{kV}$. The Fuji BAS-TR imaging plate (IP) detectors are calibrated to protons and carbons using the material published in Refs. [46,47], respectively.

Ion acceleration was investigated from two types of microstructured targets: microtubes and micropillars. The microtube arrays (shown framed in red in Fig. 1) are 3D printed by General Atomics using the two-photon polymerization (2PP) additive manufacturing technique. The microtubes are made of a polymer $\mathrm{C}_{14} \mathrm{H}_{18} \mathrm{O}_{7}$ with a mass density of $1.18 \mathrm{~g} / \mathrm{cm}^{3}$ and can be printed over large arrays of several hundreds of microns. The micropillars (shown framed in yellow in Fig. 1) are formed by Colorado State University by Co electroplating into porous alumina membranes which were subsequently dissolved [21].

\section{RESULTS}

For laser intensity of $\sim 1 \times 10^{21} \mathrm{~W} / \mathrm{cm}^{2}$ on a $1-\mu \mathrm{m}$ substrate thickness, the main ion acceleration mechanism remains target normal sheath acceleration (TNSA), in which a sheath field (of the order of $\mathrm{TV} / \mathrm{m}$ ) develops at the nonirradiated side of the target $[19,48]$. Atoms located in the substrate and in 

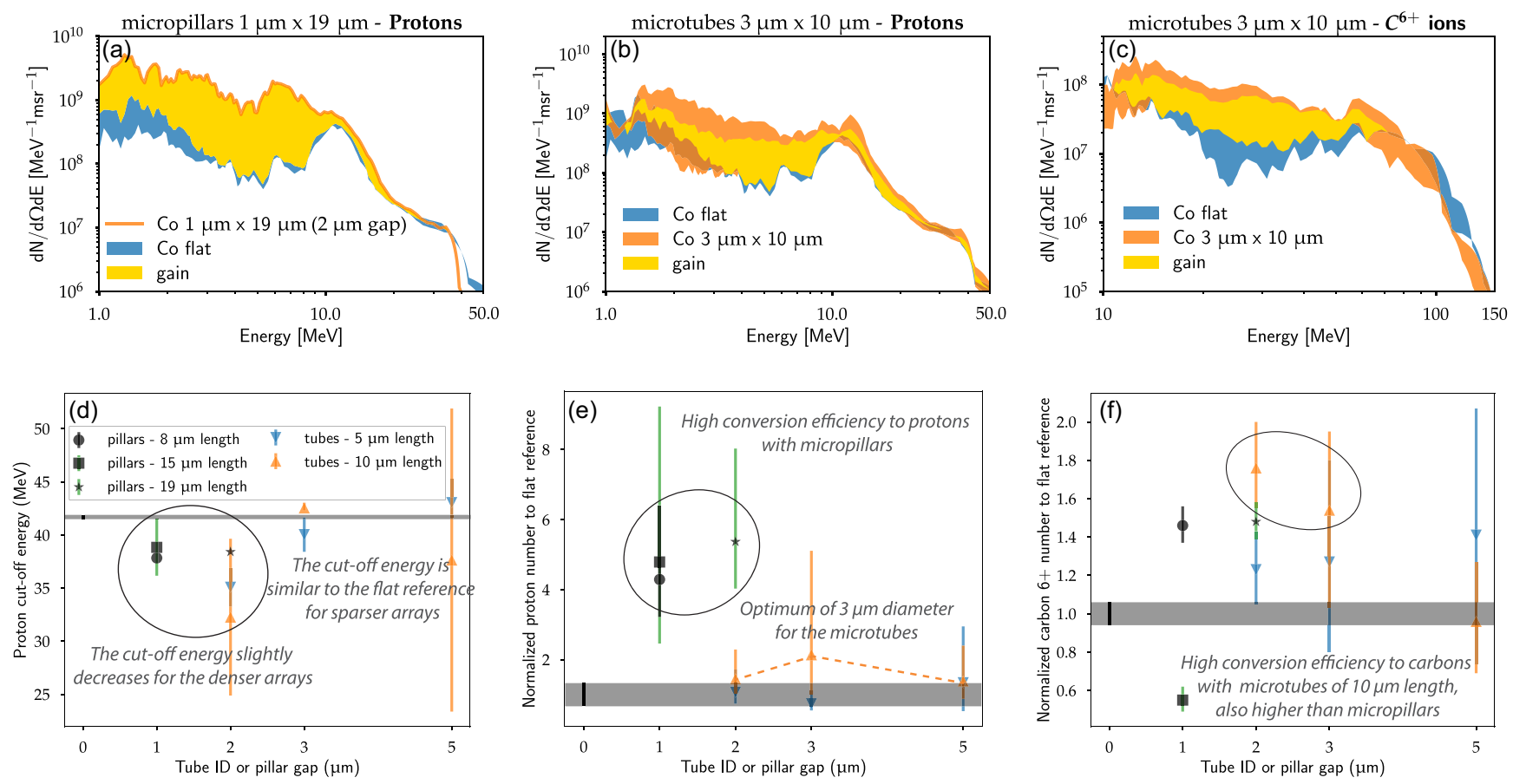

FIG. 2. (a)-(c) Ion energy distribution along target normal measured by the TP diagnostic for flat 1- $\mu$ m-thick Co foils and structured foils. In the notation $d \times h, d$ is the tube inner diameter or pillar diameter and $h$ the tube or pillar height. The blue and orange bands indicate the full range observed within all shots on the same target type in each energy bin for the flat reference and structured target, respectively. The yellow shading is the gain from flat to structured targets taken from the average spectra. (a) Proton spectrum from flat references and a micropillar target. (b), (c) Ion spectra from microtube targets, respectively (b) for protons and (c) for carbon-6+ ions. (d) plots the proton cutoff energy for the different types of targets. (e)/(f) show the total proton/carbon number enhancement for the different types of targets, normalized to the flat reference. The gray bands in each plot represent the full range variation $(\mathrm{min} / \mathrm{max})$ of the flat reference. The flat reference variations are taken into account in the error propagation when calculating the error bars ( $\mathrm{min} / \mathrm{max}$ ) for the normalized plots (e) and (f). The number of shots used is 2 for the flat reference; 1 for the 8 - $\mu$ m-length pillars; 2 for the 15 - $\mu$ m-length pillars; 1 for the $19-\mu \mathrm{m}$-length pillars; 2 for the $2 \mu \mathrm{m} \times 5 \mu \mathrm{m}$, $3 \mu \mathrm{m} \times 5 \mu \mathrm{m}, 2 \mu \mathrm{m} \times 10 \mu \mathrm{m}$, and $5 \mu \mathrm{m} \times 10 \mu \mathrm{m}$ tubes; and 3 for the $5 \mu \mathrm{m} \times 5 \mu \mathrm{m}$ and $3 \mu \mathrm{m} \times 10 \mu \mathrm{m}$ tubes.

surface layers, which are formed by contaminants of hydrocarbons and $\mathrm{H}_{2} \mathrm{O}$, become rapidly ionized and subsequently accelerated. The ions accelerated by TNSA are distributed over a broad continuous exponential energy spread, with a cutoff energy which depends on the peak strength of the sheath. A cutoff proton energy of $\sim 40-50 \mathrm{MeV}$ was recorded without a clear dependence on the target type.

The first row of Fig. 2 shows the proton and carbon-6+ energy distributions measured by the TP for selected micropillar and microtube targets which gave the highest proton yields. The width of the lines is representative of the shot-to-shot full-range variations $(\mathrm{min} / \max )$ for the flat reference in blue and for the structured target in orange. The yellow shading is the gain from flat to structured targets taken from the average spectra. The second row of Fig. 2 incorporates all the targets explored during the experiment, with Fig. 2(d) showing the proton cutoff energy and Fig. 2(e) [Fig. 2(f)] showing the total proton [carbon] number normalized to the flat reference. To plot microtubes and micropillars together on the graphs, we conflate the average gap size of micropillars and the inner diameter of the tubes, since in both cases it represents the periodic vacuum space in between the structures' walls. The highest proton number yield is obtained from micropillars of $1 \mu \mathrm{m}$ diameter and $19 \mu \mathrm{m}$ height, separated by $\approx 2 \mu \mathrm{m}$. For this target, the proton yield was $\approx 5 \times$ higher than for the flat reference. Figures 2(e) and 2(f) show that the highest proton numbers were obtained from micropillar targets, while the highest carbon numbers were obtained from microtube targets, with an increase of carbon ions of $\approx 2 \times$ in this latter case. The micropillar target scan is too limited to determine the respective roles of height and pillar gap size on the ion acceleration. However, a clear trend appears for the microtubes, showing an optimum for the $3 \mu \mathrm{m}$ diameter. Indeed, both the proton numbers and cutoff energies increase from 2 to $3 \mu \mathrm{m}$ diameter and decrease again for $5 \mu \mathrm{m}$ diameter. It is also clear here that the amount of ions measured along the target normal are higher for the tubes of $10 \mu \mathrm{m}$ heights compared to $5 \mu \mathrm{m}$ heights.

To estimate the energy conversion efficiency from the laser to accelerated protons, we assume that the proton beam at the detector has a uniform spatial distribution within a half angle $\theta$ given by the empirical law found in Ref. [49] (Gaussian laser spot's case), which takes into account the reduced divergence at higher proton energies. The empirical law writes $\theta_{E_{p} / E_{p, \text { max }}}=a_{2}\left(E_{p} / E_{p, \text { max }}\right)^{2}+a_{1}\left(E_{p} / E_{p, \text { max }}\right)+a_{0}$ with $a_{2}=-25.07, a_{1}=0.33$, and $a_{0}=31.25$. In our case, $E_{p, \max }=42 \mathrm{MeV}$ [see Fig. 2(d)]. The conversion efficiency amounts to $\approx 7 \%(\approx 11 \mathrm{~J}$ in the proton beam $)$ for the best structured targets, while it is $2.8 \pm 1 \%$ for flat references. This high conversion efficiency is at the top end of reported values 
for TNSA in the literature [18,50] and would correspond to a situation where the contaminant layer is most likely depleted [51]. It is worth noting that this conversion efficiency is nearly the same for the best micropillar $(7.4 \pm 1 \%)$ and microtube targets $(6 \pm 1 \%)$ : While the micropillar target tends to predominantly increase the low-energy protons with a slightly reduced cutoff energy [Fig. 2(a)], the microtube target yielded a gain until the highest proton energies, without showing a cutoff reduction [Fig. 2(b)].

To investigate the role of the front structures on the cutoff energy and numbers of accelerated ions, we ran simulations using the 2D particle-in-cell (PIC) code EPIC [52,53], which includes collisional relaxation and ionization processes. With the laser parameters of the experiment, we simulated the interaction of the PHELIX laser with (a) a flat $1-\mu \mathrm{m}$ Co foil, with (b) a structured $3 \mu \mathrm{m} \times 10 \mu \mathrm{m}$ microtube target, and with (c) a structured micropillar target with $d=1 \mu \mathrm{m}, h=8 \mu \mathrm{m}$, and a gap of $1 \mu \mathrm{m}$. The simulation box size was $L_{x} \times L_{z}=$ $52 \mu \mathrm{m} \times 80 \mu \mathrm{m}$ (the mesh number was $N_{x} \times N_{z}=2600 \times$ 4000 , i.e., a mesh size of $\Delta_{x, z}=0.02 \mu \mathrm{m}$ ), with $z$ and $x$ being the longitudinal and transverse directions, respectively. The laser in the simulations was Gaussian in time and space (5 $\mu \mathrm{m}$ and $0.5 \mathrm{ps}$ FWHM, respectively) and linearly polarized in the $(x, z)$ plane. The time step was chosen so as to have the Courant-Friedrichs-Lewy (CFL) condition $c \Delta t / \Delta_{x, z}=0.2$. We initialized the target with equal ion and electron densities. The initial density for the pillars and the foil substrate was the Co solid density $n_{\mathrm{Co}}=9.1 \times 10^{22} \mathrm{~cm}^{-3}$. For the tubes, the ion species was defined so as to have the same fully ionized electron density as fully ionized $\mathrm{CH}$ with $1.18 \mathrm{~g} / \mathrm{cm}^{3}$ initial mass density, i.e., $n=3.81 \times 10^{23} / Z \mathrm{~cm}^{-3}$. The atomic number $Z$ was chosen to be 14 (Si) in order to discriminate the tube's ions from the carbon and protons from the contaminant layer, but note that the tubes will be rapidly fully ionized by the laser field and get to the same density as the fully ionized $\mathrm{CH}$ of the same initial mass density as in the experiment. A contaminant layer was added at the rear of the film with a thickness of $20 \mathrm{~nm}$, assumed to be composed of carbon ions and protons at equal densities $n_{\mathrm{H}}=n_{\mathrm{C}}=1.15 \times 10^{22} \mathrm{~cm}^{-3}$; the sum corresponds to the total atom density of $\mathrm{CH}$ with a mass density of $0.5 \mathrm{~g} / \mathrm{cm}^{3}$. The mass density is set to match an equivalent areal density for a 10-nm-thick $\mathrm{CH}$ layer at $1 \mathrm{~g} / \mathrm{cm}^{3}$. The particle weight was equal for all species and there were 300 ions/mesh for Co, 90 ions/mesh for $\mathrm{Si}$, and 76 ions/mesh for $\mathrm{C}$ and $\mathrm{H}$. The initial temperatures were $T_{i}=T_{e}=100 \mathrm{eV}$, which quickly increased due to laser heating. The resulting laser electric field (on the target front) and ion densities (on the target rear) are displayed in Figs. 3(a)-3(c). For both targets, the cutoff energy is similar to (or slightly lower than) that of the flat reference. This trend is also clear in our experimental results. Note that the 2D PIC simulations tend to overestimate the ion cutoff energies [54] and a quantitative comparison of the absolute cutoff value would ideally require $3 \mathrm{D}$ simulations. Yet, the 2D PIC simulations could capture the relative cutoff energies of the different target designs and satisfactorily reproduce the tendency of the experiments. We ran more simulations with microtube targets matching those used in the experiment. The results are summarized in Figs. 3(d) and 3(e), respectively showing the proton and carbon numbers normalized to the flat

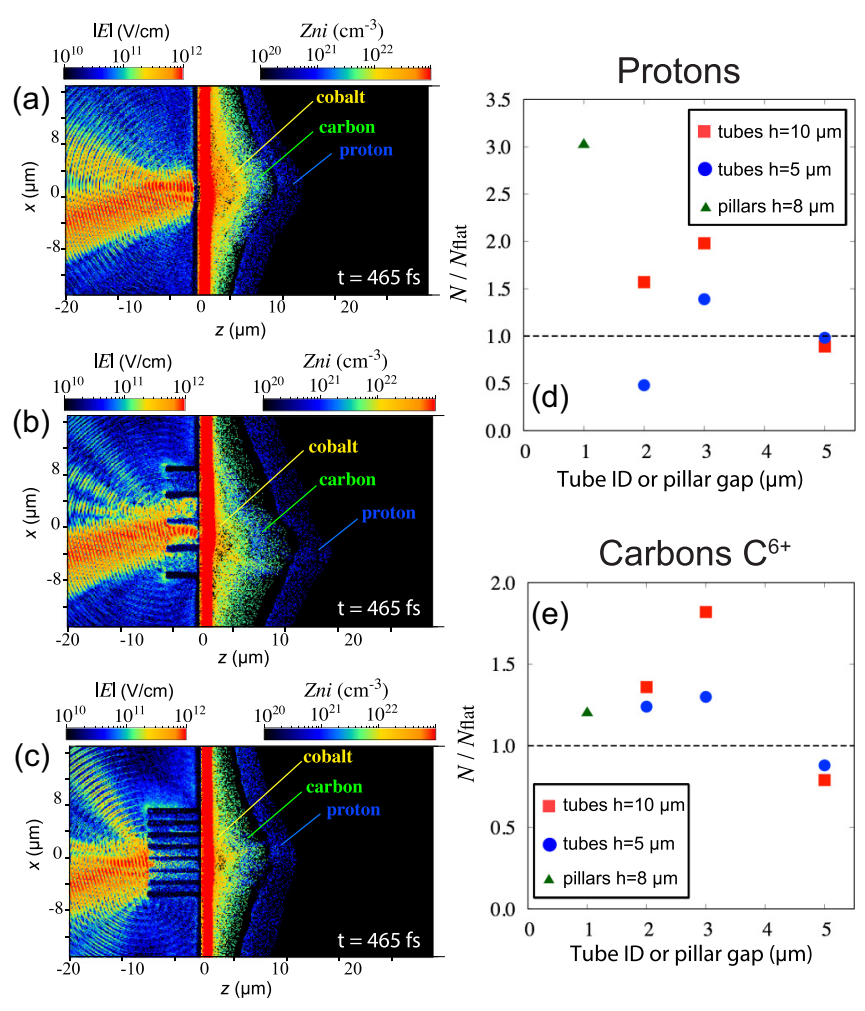

FIG. 3. Ion acceleration results from the 2D PIC simulations. Laser electric field (front side) and ion densities (rear side) at $t=465 \mathrm{fs}(\approx 100 \mathrm{fs}$ before the laser peak reaches the substrate) for (a) a flat reference, (b) a $3 \mu \mathrm{m} \times 5 \mu \mathrm{m}$ microtube target, and (c) a $1 \mu \mathrm{m} \times 8 \mu \mathrm{m}$ micropillar target with an average gap size of $1 \mu \mathrm{m}$. In (d) and (e) are shown respectively the proton and carbon signals normalized to the flat reference (black dashed line), for all the microtube targets explored during the experiment and for the pillar target of (c).

reference, as done previously in Figs. 2(e) and 2(f) with the experimental data. Analogous conclusions can be drawn here, with an optimum for the $3 \mu \mathrm{m}$ diameter and higher numbers of protons and carbons obtained for the $10 \mu \mathrm{m}$ heights' tubes. Moreover, the pillar targets also show greater proton numbers compared to microtubes, while conversely the microtubes produce greater numbers of carbon ions.

The increase of total particle numbers from the structured targets is attributed to increased laser absorption efficiency. The additional electrons accelerated from the tube walls eventually contribute to the sheath field when they reach the rear surface of the substrate. This is seen in Fig. 4(a), which shows the energy balance from fields (black curve), ions (red curve), and electrons (blue curve), for the flat (solid) and $3 \mu \mathrm{m} \times$ $5 \mu \mathrm{m}$ microtube target (dashed). In the case of the structured target, the field energy is more efficiently transferred to electrons and in the same proportion to ions, as indicated by the encircled ion energy at $1.2 \mathrm{ps}$ (end of the simulation).

In Fig. 4(b), we show the time evolution of the average electron density over the midplane of the tube for the different tube diameters and heights. The horizontal dashed line corresponds to the relativistically corrected electron density and represents a threshold for the transparency of the laser into 

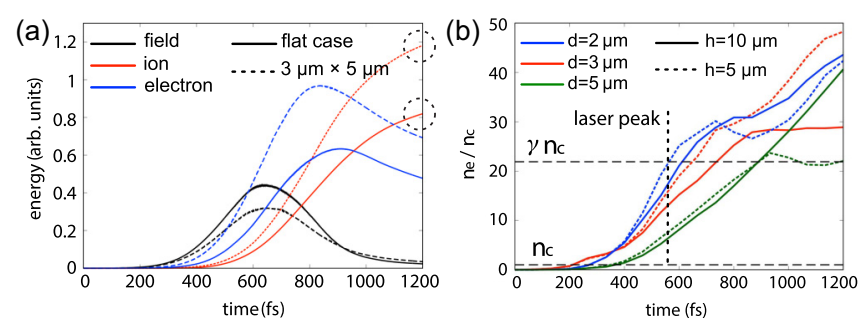

FIG. 4. (a) Energy balance, divided between fields (black), ions (red), and electrons (blue) for the flat reference (solid line) and a $3 \mu \mathrm{m} \times 5 \mu \mathrm{m}$ microtube structured target (dashed line). (b) Density normalized to the critical density for the different microtubes' diameters $(2 \mu \mathrm{m}$ in blue, $3 \mu \mathrm{m}$ in red, and $5 \mu \mathrm{m}$ in green) and heights $(10 \mu \mathrm{m}$ in solid lines and $5 \mu \mathrm{m}$ in dashed lines). The horizontal dashed line corresponds to the relativistically corrected critical density $\gamma n_{\mathrm{c}}$ and the vertical dashed line to the instant when the laser peak reaches the substrate.

the plasma. We can observe that the plasma filling the tube exceeds $n_{c}$ close to the laser peak instant $(t \approx 569 \mathrm{fs})$ and even exceeds $\gamma n_{\mathrm{c}}$ for the $d=2 \mu \mathrm{m}$ case, which would shutter the laser completely. Therefore, the electrons of maximum energy produced at the laser peak are created far from the substrate rear, and as a consequence the sheath field peak value and ion cutoff energy are reduced compared to the flat case. For pillars, due to the equivalent higher density of the structures, this plasma shuttering would happen even earlier. As the acceleration of carbons takes more time to be triggered compared to protons, due to their additional ionization time, it is thus not surprising to see that the carbons are less efficiently accelerated from the micropillars with respect to the microtubes. The plasma expansion of the tubes for laser pulses of $\gtrsim 200 \mathrm{fs}$ length is therefore a limitation for the increase of ion cutoff energies from high-energy and tightly focused laser beams with structured targets. Nevertheless, before the laser is effectively shuttered by the plasma, a larger number of free electrons are generated from the structures, thus leading to a larger number of accelerated ions.

\section{SUMMARY}

In summary, this Rapid Communication presented an experimental and computational study of the performance and limitation of two different types of front-surface microstructures for enhanced TNSA in the case of a highenergy and high-intensity (tightly focused) laser driver. The main experimental observations are as follows: (i) a net increase of the number of accelerated protons (up to $5 \times$ ) and carbons (up to $2 \times$ ) for structured targets with respect to flat references, (ii) a proton cutoff energy similar to or slightly reduced for structured targets with respect to flat references, and (iii) an optimum for a microtube diameter of $3 \mu \mathrm{m}$ (for a focal spot of $5 \mu \mathrm{m}$ FWHM). The results are reproduced using 2D PIC simulations, which highlight the role of the plasma expansion between the solid structures, resulting in higher laser absorption and a high laser-to-proton conversion efficiency up to $\approx 7 \%$. However, this plasma infill shuttered the laser within the structure relief, pulling the electron source back from the target rear, resulting in no net cutoff energy increase. For small gap dimensions, the laser shuttering can occur before the laser peak. This is especially relevant for the denser micropillars which produce a higher number of low-energy protons $(<10 \mathrm{MeV})$ relatively to microtubes, for which the proton numbers increase across the entire spectrum and more carbon ions are accelerated.

By employing a shorter laser pulse duration $(\leqslant 200 \mathrm{fs})$ at high contrast, the cutoff energies of accelerated ions would increase compared to their respective flat reference, due to laser self-focusing (especially at $I_{0}>6 \times 10^{21} \mathrm{~W} / \mathrm{cm}^{2}$ [27]) and preacceleration of electrons in the tube, although at the expense of a lower enhancement in terms of ion numbers. The optimum for the tube diameter could deviate from the studied case of this work and warrants further measurements and simulations in future works.

The data that support the findings of this study are available from the corresponding author upon reasonable request.

\section{ACKNOWLEDGMENTS}

We would like to thank the PHELIX laser team for their invaluable support for the execution of the experiment. This work is partially supported by the Department of Energy, National Nuclear Security Administration under Award No. DE-NA0003842 and by the University of California Office of the President Lab Fee under Grant No. LFR-17-449059. The work at Colorado State University was supported by the Air Force Office of Scientific Research under Award No. FA9550-17-1-0278. This work used the Extreme Science and Engineering Discovery Environment (XSEDE [55]), which is supported by National Science Foundation Grant No. ACI-1548562, SDSC Dell Cluster with Intel Haswell Processors (Comet) at the service-provider through allocation PHY180053. The microtube target fabrication and characterization at General Atomics was supported by its internal research and development funds.
[1] G. A. Mourou, T. Tajima, and S. V. Bulanov, Rev. Mod. Phys. 78, 309 (2006).

[2] A. Macchi, M. Borghesi, and M. Passoni, Rev. Mod. Phys. 85, 751 (2013).

[3] K. W. D. Ledingham, Hyperfine Interact. 171, 69 (2006).

[4] B. A. Remington, Plasma Phys. Controlled Fusion 47, A191 (2005).
[5] M. Koenig, A. Benuzzi-Mounaix, E. Brambrink, A. Nourou, A. Ravasio, H. G. Wei, T. Vinci, S. Mazevet, F. Occelli, G. Morard, F. Guyot, T. De Resseguier, and E. Lescoute, High Energy Density Phys. 6, 210 (2010).

[6] B. M. Hegelich, D. Jung, B. J. Albright, J. C. Fernandez, D. C. Gautier, C. Huang, T. J. Kwan, S. Letzring, S. Palaniyappan, R. C. Shah, H. C. Wu, L. Yin, A. Henig, R. Hörlein, D. Kiefer, 
J. Schreiber, X. Q. Yan, T. Tajima, D. Habs, B. Dromey, and J. J. Honrubia, Nucl. Fusion 51, 083011 (2011).

[7] V. Malka, Med. Phys. 31, 1587 (2004).

[8] K. W. D. Ledingham, P. McKenna, T. McCanny, S. Shimizu, J. M. Yang, L. Robson, J. Zweit, J. M. Gillies, J. Bailey, G. N. Chimon, R. J. Clarke, D. Neely, P. A. Norreys, J. L. Collier, R. P. Singhal, M. S. Wei, S. P. D. Mangles, P. Nilson, K. Krushelnick, and M. Zepf, J. Phys. D: Appl. Phys. 37, 2341 (2004).

[9] S. V. Bulanov, J. J. Wilkens, T. Z. Esirkepov, G. Korn, G. Kraft, S. D. Kraft, M. Molls, and V. S. Khoroshkov, Phys. Usp. 57, 1149 (2014).

[10] A. Maksimchuk, A. Raymond, F. Yu, G. M. Petrov, F. Dollar, L. Willingale, C. Zulick, J. Davis, and K. Krushelnick, Appl. Phys. Lett. 102, 191117 (2013).

[11] M. Borghesi, S. V. Bulanov, T. Z. Esirkepov, S. Fritzler, S. Kar, T. V. Liseikina, V. Malka, F. Pegoraro, L. Romagnani, J. P. Rousseau, A. Schiavi, O. Willi, and A. V. Zayats, Phys. Rev. Lett. 94, 195003 (2005).

[12] L. Romagnani, J. Fuchs, M. Borghesi, P. Antici, P. Audebert, F. Ceccherini, T. Cowan, T. Grismayer, S. Kar, A. Macchi, P. Mora, G. Pretzler, A. Schiavi, T. Toncian, and O. Willi, Phys. Rev. Lett. 95, 195001 (2005).

[13] J. R. Rygg, F. H. Séguin, C. K. Li, J. A. Frenje, M. J. E. Manuel, R. D. Petrasso, R. Betti, J. A. Delettrez, O. V. Gotchev, J. P. Knauer, D. D. Meyerhofer, F. J. Marshall, C. Stoeckl, and W. Theobald, Science 319, 1223 (2008).

[14] M. Borghesi, D. H. Campbell, A. Schiavi, M. G. Haines, O. Willi, A. J. MacKinnon, P. Patel, L. A. Gizzi, M. Galimberti, R. J. Clarke, F. Pegoraro, H. Ruhl, and S. Bulanov, Phys. Plasmas 9, 2214 (2002).

[15] A. J. Mackinnon, P. K. Patel, R. P. Town, M. J. Edwards, T. Phillips, S. C. Lerner, D. W. Price, D. Hicks, M. H. Key, S. Hatchett, S. C. Wilks, M. Borghesi, L. Romagnani, S. Kar, T. Toncian, G. Pretzler, O. Willi, M. Koenig, E. Martinolli, S. Lepape et al., Rev. Sci. Instrum. 75, 3531 (2004).

[16] J. J. Santos, M. Bailly-Grandvaux, L. Giuffrida, P. ForestierColleoni, S. Fujioka, Z. Zhang, P. Korneev, R. Bouillaud, S. Dorard, D. Batani, M. Chevrot, J. E. Cross, R. Crowston, J.-L. Dubois, J. Gazave, G. Gregori, E. d'Humières, S. Hulin, K. Ishihara, S. Kojima et al., New J. Phys. 17, 083051 (2015).

[17] J. Fuchs, P. Antici, E. D’Humières, E. Lefebvre, M. Borghesi, E. Brambrink, C. A. Cecchetti, M. Kaluza, V. Malka, M. Manclossi, S. Meyroneinc, P. Mora, J. Schreiber, T. Toncian, H. Pépin, and P. Audebert, Nat. Phys. 2, 48 (2006).

[18] M. Roth and M. Schollmeier, CERN Yellow Rep. 1, 231 (2016).

[19] S. C. Wilks, A. B. Langdon, T. E. Cowan, M. Roth, M. Singh, S. Hatchett, M. H. Key, D. Pennington, A. MacKinnon, and R. A. Snavely, Phys. Plasmas 8, 542 (2001).

[20] J. Li, A. V. Arefiev, S. S. Bulanov, D. Kawahito, M. BaillyGrandvaux, G. M. Petrov, C. McGuffey, and F. N. Beg, Sci. Rep. 9, 666 - Supplementary Material (2019).

[21] M. A. Purvis, V. N. Shlyaptsev, R. Hollinger, C. Bargsten, A. Pukhov, A. Prieto, Y. Wang, B. M. Luther, L. Yin, S. Wang, and J. J. Rocca, Nat. Photonics 7, 796 (2013).

[22] D. Margarone, O. Klimo, I. J. Kim, J. Prokůpek, J. Limpouch, T. M. Jeong, T. Mocek, J. Pšikal, H. T. Kim, J. Proška, K. H. Nam, L. Štolcová, I. W. Choi, S. K. Lee, J. H. Sung, T. J. Yu, and G. Korn, Phys. Rev. Lett. 109, 234801 (2012).

[23] S. Jiang, A. G. Krygier, D. W. Schumacher, K. U. Akli, and R. R. Freeman, Phys. Rev. E 89, 013106 (2014).
[24] S. Jiang, L. L. Ji, H. Audesirk, K. M. George, J. Snyder, A. Krygier, P. Poole, C. Willis, R. Daskalova, E. Chowdhury, N. S. Lewis, D. W. Schumacher, A. Pukhov, R. R. Freeman, and K. U. Akli, Phys. Rev. Lett. 116, 085002 (2016).

[25] M. Blanco, M. T. Flores-Arias, C. Ruiz, and M. Vranic, New J. Phys. 19, 033004 (2017).

[26] L. L. Ji, J. Snyder, A. Pukhov, R. R. Freeman, and K. U. Akli, Sci. Rep. 6, 23256 (2016).

[27] L. Ji, S. Jiang, A. Pukhov, R. Freeman, and K. Akli, High Power Laser Sci. Eng. 5, e14 (2017).

[28] Z. Zhao, L. Cao, L. Cao, J. Wang, W. Huang, W. Jiang, Y. He, Y. Wu, B. Zhu, K. Dong, Y. Ding, B. Zhang, Y. Gu, M. Y. Yu, and X. T. He, Phys. Plasmas 17, 123108 (2010).

[29] T. Ceccotti, V. Floquet, A. Sgattoni, A. Bigongiari, O. Klimo, M. Raynaud, C. Riconda, A. Heron, F. Baffigi, L. Labate, L. A. Gizzi, L. Vassura, J. Fuchs, M. Passoni, M. Květon, F. Novotny, M. Possolt, J. Prokůpek, J. Proška, J. Pšikal, L. Štolcová, A. Velyhan, M. Bougeard, P. D’Oliveira, O. Tcherbakoff, F. Réau, P. Martin, and A. Macchi, Phys. Rev. Lett. 111, 185001 (2013).

[30] D. Khaghani, M. Lobet, B. Borm, L. Burr, F. Gärtner, L. Gremillet, L. Movsesyan, O. Rosmej, M. E. ToimilMolares, F. Wagner, and P. Neumayer, Sci. Rep. 7, 11366 (2017).

[31] J. Snyder, L. L. Ji, K. M. George, C. Willis, G. E. Cochran, R. L. Daskalova, A. Handler, T. Rubin, P. L. Poole, D. Nasir, A. Zingale, E. Chowdhury, B. F. Shen, and D. W. Schumacher, Phys. Plasmas 26, 033110 (2019).

[32] M. V. Sedov, A. Y. Faenov, A. A. Andreev, I. Y. Skobelev, S. N. Ryazantsev, T. A. Pikuz, P. Durey, L. Doehl, D. Farley, C. D. Baird, K. L. Lancaster, C. D. Murphy, N. Booth, C. Spindloe, K. Y. Platonov, P. McKenna, R. Kodama, N. Woolsey, and S. A. Pikuz, Laser Part. Beams 37, 176 (2019).

[33] A. Moreau, R. Hollinger, C. Calvi, S. Wang, Y. Wang, M. G. Capeluto, A. Rockwood, A. Curtis, S. Kasdorf, V. N. Shlyaptsev, V. Kaymak, A. Pukhov, and J. J. Rocca, Plasma Phys. Controlled Fusion 62, 014013 (2020).

[34] O. Klimo, J. Psikal, J. Limpouch, J. Proska, F. Novotny, T. Ceccotti, V. Floquet, and S. Kawata, New J. Phys. 13, 053028 (2011).

[35] A. Bigongiari, M. Raynaud, C. Riconda, and A. Héron, Phys. Plasmas 20, 052701 (2013).

[36] D. B. Zou, A. Pukhov, L. Q. Yi, H. B. Zhou, T. P. Yu, Y. Yin, and F. Q. Shao, Sci. Rep. 7, 42666 (2017).

[37] B. Feng, L. L. Ji, B. F. Shen, X. S. Geng, Z. Guo, Q. Yu, T. J. Xu, and L. G. Zhang, Phys. Plasmas 25, 103109 (2018).

[38] A. Curtis, C. Calvi, J. Tinsley, R. Hollinger, V. Kaymak, A. Pukhov, S. Wang, A. Rockwood, Y. Wang, V. N. Shlyaptsev, and J. J. Rocca, Nat. Commun. 9, 1077 (2018).

[39] S. Mondal, I. Chakraborty, S. Ahmad, D. Carvalho, P. Singh, A. D. Lad, V. Narayanan, P. Ayyub, G. R. Kumar, J. Zheng, and Z. M. Sheng, Phys. Rev. B 83, 035408 (2011).

[40] L. Yi, A. Pukhov, P. Luu-Thanh, and B. Shen, Phys. Rev. Lett. 116, 115001 (2016).

[41] Z. Lécz and A. Andreev, Phys. Plasmas 24, 033113 (2017).

[42] R. Hollinger, C. Bargsten, V. N. Shlyaptsev, V. Kaymak, A. e. Pukhov, M. G. Capeluto, S. Wang, A. Rockwood, Y. Wang, A. Townsend, A. Prieto, P. Stockton, A. Curtis, and J. J. Rocca, Optica 4, 1344 (2017).

[43] D. Rolles, Nat. Photonics 12, 62 (2018). 
[44] S. Mondal, Q. Wei, W. J. Ding, H. A. Hafez, M. A. Fareed, A. Laramée, X. Ropagnol, G. Zhang, S. Sun, Z. M. Sheng, J. Zhang, and T. Ozaki, Sci. Rep. 7, 40058 (2017).

[45] D. C. Carroll, P. Brummitt, D. Neely, F. Lindau, O. Lundh, C. G. Wahlström, and P. McKenna, Nucl. Instrum. Methods Phys. Res. A 620, 23 (2010).

[46] N. Rabhi, D. Batani, G. Boutoux, J. E. Ducret, K. Jakubowska, I. Lantuejoul-Thfoin, C. Nauraye, A. Patriarca, A. Saïd, A. Semsoum, L. Serani, B. Thomas, and B. Vauzour, Rev. Sci. Instrum. 88, 113301 (2017).

[47] D. Doria, S. Kar, H. Ahmed, A. Alejo, J. Fernand ez, M. Cerchez, R. J. Gray, F. Hanton, D. A. MacLellan, P. McKenna, Z. Najmudin, D. Neely, L. Romagnani, J. A. Ruiz, G. Sarri, C. Scullion, M. Streeter, M. Swantusch, O. Willi, M. Zepf, and M. Borghesi, Rev. Sci. Instrum. 86, 123302 (2015).

[48] P. Mora, Phys. Rev. Lett. 90, 185002 (2003).

[49] C. Brabetz, S. Busold, T. Cowan, O. Deppert, D. Jahn, O. Kester, M. Roth, D. Schumacher, and V. Bagnoud, Phys. Plasmas 22, 013105 (2015).
[50] C. M. Brenner, A. P. L. Robinson, K. Markey, R. H. H. Scott, R. J. Gray, M. Rosinski, O. Deppert, J. Badziak, D. Batani, J. R. Davies, S. M. Hassan, K. L. Lancaster, K. Li, I. O. Musgrave, P. A. Norreys, J. Pasley, M. Roth, H. P. Schlenvoigt, C. Spindloe, M. Tatarakis et al., Appl. Phys. Lett. 104, 081123 (2014).

[51] M. Allen, P. K. Patel, A. Mackinnon, D. Price, S. Wilks, and E. Morse, Phys. Rev. Lett. 93, 265004 (2004).

[52] D. Kawahito and Y. Kishimoto, Phys. Plasmas 24, 103105 (2017).

[53] D. Kawahito and Y. Kishimoto, Phys. Plasmas 27, 033108 (2020).

[54] A. Héron, J. C. Adam, and P. Mora, Phys. Plasmas 27, 013103 (2020).

[55] J. Towns, T. Cockerill, M. Dahan, I. Foster, K. Gaither, A. Grimshaw, V. Hazlewood, S. Lathrop, D. Lifka, G. D. Peterson, R. Roskies, J. R. Scott, and N. Wilkens-Diehr, Comput. Sci. Eng. 16, 62 (2014). 